\title{
Erwiderung auf den Leserbrief zu Lipoedema - myths and facts Part 1 and 5. European Best Practice of Lipoedema - Summary of the European Lipoedema Consensus, Phlebologie 2020; 49: 31-49
}

Der Leserbrief aus den USA freut uns, zeigt er doch, dass unsere Artikelserie über das Lipödem nicht nur deutschland- und europaweit gelesen wird. Gut 21/2 Jahre nach Veröffentlichung von Teil 1 befinden sich alle 5 Publikationen unter den Top 10 der „meistgelesenen Artikel“ in der Phlebologie (Stand August 2020) [1]. Auch in Europa haben die „Mythen und Fakten zum Lipödem “ eine hohe Resonanz erfahren und tragen weiter zum dringend notwendigen Paradigmenwechsel dieser Erkrankung bei. Renommierte Lipödem-Experten aus 10 europäischen Ländern unterstützen inzwischen den im fünften Teil der Artikelserie erarbeiteten Konsensus zum Lipödem [2]. In „Pathways“, dem offiziellen Magazin des Canadian Lymphedema Framework, werden sowohl die Mythen des Lipödems als auch der Paradigmenwechsel das Titelthema in der Herbst-Ausgabe sein.

Dass in den USA weiter am alten Narrativ dieser Erkrankung festgehalten wird, ist uns bewusst. Dort dominieren 3 in den Medien stark vertretene und finanzkräftige Patientenorganisationen sowie wenige eng mit diesen Organisationen verbundene Ärzte das Meinungsbild zum Lipödem: Lipedema Simplified von Catherine Seo [3], Fat Disorder Research Society (FDRS) [4] und Lipedema Foundation [5], wobei die beiden letztgenannten eng miteinander verwoben sind. Felicitie Daftuar ist sowohl im Leadership Team von FDRS [6] als auch Gründerin und Executive Director von Lipedema Foundation [7]. Dies ist wichtig zu wissen, da die Verfasserin des Leserbriefs - Karen Herbst - sehr eng sowohl mit FDRS [8] als auch mit Lipedema Foundation [9] verbunden ist. Seit 2015 unterstützt Felicitie Daftuar mit Millionenbeträgen Projekte von Karen Herbst [10, 11].

Bevor wir auf die Mitunterzeichner des Leserbriefs eingehen, werden wir die strittigen Punkte A bis F inhaltlich klären.

Die Leserbriefautorin beginnt ihr Schreiben mit der Feststellung: „Lipedema is a disease of loose connective tissue (LCT) not just fat. Constituents of LCT include cells and an extracellular matrix of collagen fibers around glycosaminoglycans (GAGs) bound to sodium and water."

Für das Statement, Lipödem sei eine Erkrankung des lockeren Bindegewebes, existiert kein wissenschaftlicher Nachweis; eine entsprechende wissenschaftliche Referenz für diese Behauptung wird auch erst gar nicht von der Leserbriefautorin genannt. Richtig hingegen ist die Aussage, dass lockeres Bindegewebe eine Extrazellulärmatrix hat, die aus Fasern und Grundsubsubstanz (hier auch Glykosaminoglykane) besteht. Richtig ist auch, dass Glykosaminoglykane eine hohe Wasserbindungskapazität haben. Was diese Glykosaminoglykane (GAGs) nun mit dem Lipödem-Syndrom zu tun haben sollen, wird von der Leserbriefverfasserin dann unter $\mathrm{A}$ erklärt.

\section{Zu A. Edema}

So schreibt sie: „Edema is defined as excess interstitial fluid (IF) (2) that is free or bound in a GAG gel as in lipedema."

Während der erste Halbsatz dieses Statements sicher richtig ist und medizinisches Allgemeinwissen darstellt, fehlt für den zweiten Halbsatz („or bound in GAG gel as in lipedema“) jeglicher seriöse Nachweis. Als Quelle für diese Behauptung dient der Verfasserin ihre eigene unter A.2 genannte Arbeit, auf die weiter unten noch eingegangen wird.

\section{Zu 1.}

Der beschriebene „overgrowth of blood vessels in lipedema tissue" befand sich nur in der adipösen Lipödem-Studienpopulation der genannten Untersuchung von AlGhadban, nicht in der Gruppe der NichtAdipösen [12]. Somit sind die beschriebenen Veränderungen viel eher der Adipositas und nicht dem Lipödem-Syndrom zuzuschreiben. In der kürzlich veröffentlichten Arbeit von Felmerer, Hägerling und Gousopoulos bei reinen Lipödem-Patientinnen konnten lediglich vergrößerte Adipozyten (,increased adipocyte size“) festgestellt werden. Obwohl in dieser histopathologischen Untersuchung das von Hägerling entwickelte VIPAR-Verfahren der 3D-Rekonstruktion verwendet wurde, gab es keinen Hinweis auf veränderte Lymph- oder Blutgefäße bei LipödemPatientinnen [13].

Über die Ergebnisse der von der Leserbriefschreiberin erwähnten Arbeit hat der Mitautor Gousopoulos einen Vortrag auf der Lymphologie 2019 (German Congress of Lymphology) in Bad Krozingen gehalten. Sein auch im Kongress-Abstract nachlesbares Fazit lautet: „No morphological changes in the lymphatic component are present in lipoedema" [14].

Es gibt ebenfalls keinen wissenschaftlichen Nachweis für „leaky capillaries“ beim Lipödem-Syndrom. Die von der Leserbriefautorin angegebene Quelle von 1986 hatte nicht das Lipödem-Syndrom zum Gegenstand.

\section{Zu 2.-6.}

In den Punkten 2-6 wird nun regelmäßig auf das Konzept der bestehenden GAGs beim Lipödem abgehoben. Die einzige genannte Quelle, die dieses Konzept der GAGs beim Lipödem beschreiben soll, ist eine Arbeit der Leserbriefverfasserin selbst. Tiefer am Wissenschaftsverständnis von Herbst Interessierte sei diese Abhandlung („Lipedema Is Not Just Fat“) ans Herz gelegt [15]. Die von ihr dort dargelegten Behauptungen waren für uns in keiner Weise nachvollziehbar. Auch geben die von Herbst genannten Quellen keinen Hinweis darauf, was GAGs mit Lipödem zu tun haben. Zwar wird in dem von Herbst genannten Review von Reed und Rubin ausführlich auf die Glykosaminoglykane eingegangen - allerdings in einem völlig anderen Zusammenhang. In den von der Leserbriefautorin erwähnten Arbeiten, die das Lipödem zum Thema haben, kommen GAGs nicht vor, die hergestellten Bezüge zum Lipödem erscheinen uns - mit Verlaub - an den Haaren herbeigezogen. Völlig offen bleibt auch die Frage, was die 
postulierten GAGs mit der von Patientinnen angegebenen oft starken Schmerzsymptomatik zu tun haben.

Es ist daher auch nicht verwunderlich, dass dieses Konzept nicht in einem wissenschaftlichen (peer reviewed) Journal veröffentlicht wurde, sondern auf ihrer eigenen Website Lipedema.com [16], die Herbst gemeinsam mit Mitunterzeichnern und Lipödem-Aktivistinnen dieses Leserbriefs (Kahn, Iker und Ehrlich) betreibt [17]. Herbst und die gleichen Mitunterzeichner sind auch Herausgeber eines Buches (Lymphedema and Lipedema Nutrition Guide: Foods, Vitamins, Minerals and Supplements), bei dem die Vorsitzende von Lipedema Foundation, Felicitie Daftuar, das Vorwort verfasst hat [18].

Ja, wir wissen, das liest sich vielleicht ein wenig kompliziert, ist aber wichtig, um zu verstehen, wie „Wissenschaft“ in den USA funktioniert, wenn finanzstarke Patientenorganisationen - mit ganz eigenen Interessen - enge Kooperationen mit Behandlern eingehen.

\section{Zu den Fakten}

Ödeme sind definiert als „abnorme Flüssigkeitsansammlung“ im Gewebe [19]. Allerdings konnten weder klinische Untersuchungen noch Bildgebung jemals ein relevantes Ödem bei Patientinnen mit Lipödem-Syndrom nachweisen [20]. Eine multizentrische Studie mit hochauflösendem Ultraschall bei Patientinnen mit der Diagnose Lipödem fand keinen Nachweis für Flüssigkeit im Weichteilgewebe der Beine [21]. In einer 2020 erschienenen Studie, in der Patientinnen mit LipödemSyndrom mittels MR-Lymphografie untersucht wurden, konkludieren die Autoren: „The fat tissue was homogenous, without any signs of edema in pure lipedema patients" [22]. Auch in histologischen Untersuchungen wurde niemals die Präsenz eines Ödems beschrieben [20]. ReichSchupke, Altmeyer und Stücker schrieben bereits 2012 in einem wegweisenden Artikel: „Der Begriff „Lipödem“ ist eigentlich irreführend, da es sich nicht um ein Ödem, also um eine Flüssigkeitseinlagerung im Gewebe handelt“ [23]. Dies wurde auch von den Autoren der niederländischen Lipödem-Leitlinie bestätigt, indem sie den
Terminus Lipödem als unglücklich („unfortunate term“) beschreiben, da er Flüssigkeit im Gewebe suggeriert, wo keine Flüssigkeit zu finden ist [24]. Schließlich resümierte das European Lipoedema Forum - eine hochrangig besetzte internationale Expertengruppe aus 7 europäischen Ländern - in einem vielbeachteten Konsensuspapier: „There is no scientific evidence that Lipoedema is an ,oedema problem“" (Hervorhebung im Original) [25].

\section{Zu B. Manual Lymphtherapy and compression}

Neben ihrer eigenen Pilotstudie an 7 (!) Patientinnen, die mit einer „whole body manual subcutaneous adipose tissue (SAT) therapy" behandelt wurden, sind die einzigen auf das Lipödem fokussierenden Therapiestudien, die in diesem Abschnitt von der Leserbriefautorin genannt werden, die beiden Untersuchungen von Szolnoky et al. Hier untersuchten die Wissenschaftler den Effekt der komplexen physikalischen Entstauungstherapie (KPE) auf das Lipödem. KPE besteht aus manueller Lymphdrainage (MLD), Kompression, Sport- und Bewegungstherapie und Hautpflege (neuerdings auch noch additiv Selbstmanagement) [26]. Die Patientinnen dieser Studie berichteten über eine Besserung der Beschwerden unter KPE.

Die Verfasserin des Leserbriefs verwechselt nun leider MLD mit KPE. Aus der Studie von Szolnoky et al. geht nicht hervor, welche der o.g. Komponenten der KPE tatsächlich wirksam gewesen sind. Kompression und Sport sind durch ihre antiinflammatorischen Effekte tatsächlich wirksam beim Lipödem. Dies wurde in Teil 5 der Artikelserie auch so beschrieben und entspricht dem European Consensus [25]. Für die MLD, wie von der amerikanischen Verfasserin behauptet, fehlt dieser Nachweis.

Darüber hinaus, und diese Frage sei erlaubt: Wenn, wie oben dargelegt, kein Nachweis einer relevanten Flüssigkeit beim Lipödem vorliegt, welchen Sinn macht dann die immer wieder geforderte „Drainage“? Welche Substanz im Weichteilgewebe der Beine soll mit der MLD „drainiert“ werden? Fettgewebe?

\section{Zu C. Lipohypertrophy}

Die schmerz- bzw. beschwerdefreie Volumenvermehrung der Beine wird nach Herpertz [27] in den deutschsprachigen Ländern Lipohypertrophie genannt. Dies ist weitgehender Konsens und macht auch Sinn. Würde die - von der Frau subjektiv wahrgenommene - Disproportionalität der Beine bereits das Kriterium für die Diagnose Lipödem erfüllen, wäre (angesichts des gegenwärtigen Schönheitsideals, das dünne Beine präferiert und auf vielen Online-Medien propagiert wird) dem Missbrauch von Therapie jeglicher Art Tür und Tor geöffnet.

Die Verfasserin schreibt weiter: „A woman with painful lipedema who underwent therapy and has no pain, still has lipedema". Dieser Aussage stimmen wir zu. Genauso hat eine Patientin mit arterieller Hypertonie, die durch die Einnahme antihypertensiv wirksamer Medikamente jetzt normale Blutdruckwerte hat, noch immer eine arterielle Hypertonie. Allerdings - vor der Einnahme dieser Medikamente wurden vom Behandler erhöhte Blutdruckwerte gemessen. Entsprechendes gilt auch für die Beschwerden beim Lipödem-Syndrom.

\section{Zu D. Obesity, secondary lymphedema and bariatric} surgery

Selbstverständlich sind Lipödem-Syndrom, Lymphödem und Adipositas ganz unterschiedliche Krankheiten. Um diese zu differenzieren, braucht es für den auf diesem Gebiet erfahrenen Kliniker sicher keinen Biomarker. Allerdings - und hier ist die internationale Datenlage sehr konsistent sind weit über $80 \%$ der Patientinnen mit der Diagnose Lipödem auch adipös, ca. $50 \%$ sogar morbid adipös, d. h. sie haben einen BMI $\geq 40$ [28-33]. Mit anderen Worten: Lipödem und Adipositas treten bei der weit überwiegenden Mehrheit der Frauen gemeinsam auf. Morbide Adipositas kann bei manchen Menschen zu einer Lymphabflussstörung, respektive zu einem Lymphödem führen. Die Pathophysiologie hierzu ist gut belegt und wird - üblicherweise - auch nicht bestritten [34, 35]. Patienten mit Adipositas-assoziierten Lymphödemen sind die am schnellsten anstei- 
gende Patientengruppe im Europäischen Zentrum für Lymphologie in Hinterzarten.

Die Verfasserin des Leserbriefs schreibt: „Many severe obese women with lipedema do not develop lymphedema." Dieser Aussage stimmen wir zu. Allerdings auch nicht jeder „Kettenraucher“ erkrankt an einem Bronchialkarzinom. Und sicher stimmt auch die Leserbriefautorin mit uns überein, dass „Kettenrauchen“ ein deutlich erhöhtes Risiko in sich birgt, an einem Bronchialkarzinom zu erkranken.

Wir freuen uns, dass die Leserbriefverfasserin den Biomarker PF4 erwähnt, der aktuell von Stanley Rockson propagiert und in Lipödem-Patientenforen zunehmend verbreitet wird. Rockson et al. zufolge dient PF4 als Hinweis dafür, dass beim Lipödem eine lymphatische Dysfunktion vorliegt: „Finally, our results support the prevailing hypothesis, that in lipedema, lymphatic dysfunction plays a role“ [36]. In einem aktuellen Webinar von Lipedema Simplified, der anderen oben bereits erwähnten amerikanischen Lipödem-Patientenorganisation, stellt Rockson eine - vermeintliche - Lipödem-Patientin vor: „This patient has lipedema“, bei der offensichtlich PF4 erhöht war [37]. Die hier als Lipödem gezeigte Patientin leidet ganz offensichtlich an einem (wohl Adipositasassoziierten) Lymphödem mit bereits bestehender Stauungsdermatose! Wir glauben gerne, dass bei diesen Patientinnen auch PF4 erhöht ist. Die richtige Diagnose der Patientinnen ist allerdings essenziell, um valide und seriöse Ergebnisse produzieren zu können. Dies gilt auch für bildgebende Untersuchungen. Bereits ältere klinische Studien mittels indirekter Lymphografie und Lymphszintigrafie haben gezeigt, dass der Lymphtransport vom subepidermalen Kompartiment beim Lipödem eben nicht eingeschränkt ist [3840]. Auch neue Untersuchungen mit modernsten histopathologischen Untersuchungsmethoden brachten keinen Hinweis auf veränderte Lymphgefäße bei Lipödem-Patientinnen [13].

Die Datenlage zum Langzeiterfolg der bariatrischen Chirurgie bei morbider Adipositas ist sehr konsistent und überzeugend [41-49]. Eine aktuelle Untersuchung des Universitätsklinikums Freiburg gemeinsam mit dem Europäischen Zentrum für Lymphologie in Hinterzarten zum Effekt der bariatrischen Operation bei schwer adipösen Patientinnen mit Lipödem-Syndrom zeigt den guten Erfolg dieser Therapieoption und bestätigt unsere seit 2008 bestehenden Erfahrungen mit diesem Patientengut [50].

\section{Zu E. Psychology}

Auch hier widersprechen wir der Verfasserin des Leserbriefs. In einer Untersuchung an 150 Patientinnen mit der gesicherten Diagnose Lipödem-Syndrom präsentierten $80 \%$ der Frauen psychische Störungsbilder (meist Depression, Essstörung oder posttraumatische Belastungsstörung) oder eine schwere psychische Beeinträchtigung, wie z. B. Burnout oder chronische Stressbelastung. Diese Störungsbilder bzw. schwere psychische Beeinträchtigung bestanden jedoch bereits vor der Entwicklung Lipödem-assoziierter Schmerzen im Weichteilgewebe [29]. Damit kann - formallogisch - die Erkrankung Lipödem-Syndrom nicht Ursache dieser psychischen Belastung sein. Der Einfluss psychischer Belastungen und Krankheiten sowohl auf die Schmerzentstehung als auch auf die Schmerzwahrnehmung ist breit untersucht und sehr konsistent [51-57]. Depression und Essstörungen waren im Vergleich zur 12-MonatsPrävalenz dieser psychischen Störungsbilder in der Allgemeinbevölkerung [58] vor allem im 12-Monats-Zeitraum vor der Entstehung der Lipödem-assoziierten Schmerzsymptomatik stark erhöht. Daher ist das von der Leserbriefautorin kritisierte Statement „psychological factors can contribute significantly to the development of lipedema" gut belegt.

\section{Zu F. Liposuktion}

Wir wissen, dass viele Patientinnen nicht einverstanden sind, die Durchführung einer Liposuktion vom Körpergewicht (und damit auch vom Body-Mass-Index) abhängig zu machen.

Der von der Leserbriefverfasserin kritisierte European Consensus sieht den BMI von $35 \mathrm{~kg} / \mathrm{m}^{2}$ bei gleichzeitiger zentraler Adipositas als Obergrenze für die Fettabsaugung. Ab einem BMI von $35 \mathrm{~kg} / \mathrm{m}^{2}$ steht (von seltenen Ausnahmen einmal abgesehen) die Adipositas der Patientin mit Lipödem im Vordergrund - und sollte auch entsprechend fokussiert werden.
Der European Consensus empfiehlt dann die Überprüfung der Indikation für eine bariatrische OP. Dies entspricht auch den Empfehlungen der S3-Leitlinien der Deutschen Gesellschaft für Adipositas-Chirurgie. Der positive Einfluss der bariatrischen OP auf das Lipödem-Syndrom wurde inzwischen untersucht und bestätigt [50]. Die Durchführung der Liposuktion bei Patientinnen mit klarer zentraler Adipositas halten wir für einen Kunstfehler. Die Kriterien zur Liposuktion wurden im European Consensus klar definiert [2].

Auch in Deutschland führte die - weitgehend ähnliche - Einschätzung des Gemeinsamen Bundesausschusses (G-BA) zu einem Aufschrei der Entrüstung seitens der Patientenvertreter. Die Selbsthilfeverbände bezeichneten die Entscheidung des G-BA als „Farce“ [59]. In einer lesenswerten (weil demaskierenden) Presseerklärung der organisierten Selbsthilfe von Frauen mit Lipödem vom 20.09.2019 wird die Liposuktion als „rettende Behandlung“ hochstilisiert, um „ein Leben im Rollstuhl“ oder auch „eine stark verfrühte Sterblichkeit“ zu verhindern [60]. Nichts könnte weiter von der Realität entfernt sein! Weder führt das reine Lipödem (ohne begleitende schwerste Adipositas) zu einem „Leben im Rollstuhl“ noch zu einer „stark verfrühten Sterblichkeit“. Auch sind uns keine Daten bekannt, wonach die Liposuktion hinsichtlich der oben dargestellten Prognosen als „rettende Behandlung“Wirkung gezeigt hätte.

Bemerkenswert ist aber auch die Begründung der Leserbriefverfasserin für ihren Widerspruch: „We disagree that “Liposuction is not a treatment option in patients with a $B M I>35 \mathrm{~kg} / \mathrm{m}^{2}$ and central obesity $(W H t R>0.5)$ "[1]. Women can develop lipedema LCT on the abdomen [14]. "Als Quelle für dieses Lipödem des Abdomens nennt die Verfasserin wiederum eine ihrer eigenen Publikation.

Das Lipödem des Abdomens wurde bereits 2018 in einem kritischen Leserbrief von Schmeller auf die Artikelserie ins Spiel gebracht. So schrieb Schmeller: „Die Zuordnung von an Rumpf und Extremitäten lokalisierten Unterhautfettvermehrungen als Folge der Adipositas bzw. als Folge des Lipödems... kann sehr schwierig, teilweise wohl auch unmöglich sein. “ Und weiter unten:

„Ob die Adipozyten des Lipödems zusätzlich zur Subkutis der Extremitäten auch visze- 
ral im Bauchbereich eine Volumen- und Gewichtsvermehrung verursachen, kann derzeit nicht sicher beurteilt werden " [61].

Wir zitieren hier im Folgenden unsere damalige Replik auf Schmellers Gedankenwelt [62].

„Wir möchten ausdrücklich betonen, dass Unterhautfettgewebsvermehrungen am Rumpf bzw. Adipozyten im Bauchraum KEINESFALLS in einen Zusammenhang mit Lipödem gebracht werden sollten! Gedankenspiele wie diese entbehren jeglicher wissenschaftlichen Evidenz. In diesen Regionen besteht ja auch keine Schmerzsymptomatik bei den Patientinnen. Allerdings sehen wir bereits jetzt - täglich Patientinnen, die uns mit der Diagnose Lipödem (oder „Lipolymphödem“) vorgestellt werden und die fest davon überzeugt sind, dass ihr Lipödem die Ursache ihrer Gewichtszunahme, ihrer Adipositas sei. Immer häufiger sehen wir adipöse Patientinnen, die behaupten, an einem „Ganzkörperlipödem“ oder an einem „Lipödem des Bauches" zu leiden - welches ihnen auch nicht selten von ärztlichen Kollegen attestiert wird - und die hierin die Ursache ihrer Gewichtszunahme sehen. Hier möchten wir dem Autor des Leserbriefs am liebsten ganz laut zurufen: „HALT, HERR SCHMELLER, NEIN! BITTE ÖFFNEN SIE DIESE BÜCHSE DER PANDORA NICHT!“ (Zitatende).

Es scheint, als sei die Büchse inzwischen geöffnet.

Letztlich spiegelt dieser Leserbrief auch die kontroversen Debatten der zurückliegenden Jahre wider, die der Erstautor der Artikelserie mit Karen Herbst, aber auch mit Felicitie Daftuar auf Bühnen und Konferenzen in der Vergangenheit führte. Wir sind besorgt, dass die sehr verengte US-amerikanische Sicht auf das Lipödem, eine Sicht, die das Problem der Adipositas ebenso wie die - vorbestehenden - psychischen Belastungen der Frauen ignoriert, der Komplexität dieser Erkrankung nicht gerecht wird.

Noch ein abschließendes Wort zu den Mitunterzeichnern dieses Leserbriefs. Nahezu alle der dort aufgeführten Personen sind mit „The US Standard of Care Committee" verbunden. Gibt man diese vermeintliche Organisation bei Google ein, findet man keinen Eintrag. Fündig wird man allerdings auf der oben bereits erwähnten Herbst-eigenen Website „lipedema.com“, auf der die Verfasserin des
Leserbriefs, Karen Herbst, auch ihren Beitrag zu den GAGs veröffentlichten durfte. Dort wird auf ein Treffen aufmerksam gemacht, das im April 2019 in Baltimore stattfand und an dem 18 der 22 Mitunterzeichner teilgenommen hatten. Zu dieser Konferenz geladen hatten die Leserbriefautorin sowie die beiden bereits oben genannten Patientenorganisationen FDRS und Lipedema Foundation [63]. Ziel der Zusammenkunft in Baltimore war u.a. auch das Erstellen von US-Guidelines zum Lipödem. Man stelle sich diese Situation einmal in Deutschland (oder anderen europäischen Ländern) vor: LipödemSelbsthilfegruppen organisieren eine Konferenz, laden hierzu „selbstgewählte Behandler" ein und erstellen eine dann sogenannte Leitlinie.

Interessenunabhängige Forschung und Therapie, die gerade beim Lipödem essenziell wäre, sieht sicher anders aus.

Aber auch andere, nicht diesem Kreis zugehörige Mitunterzeichner, weisen engste Verbindungen zur Lipedema Foundation auf $[64,65]$.

\section{FAZIT}

Money makes the world go round das gilt auch für die von Patientinnen finanzierte und damit gesteuerte Lipödem-Forschung in den USA.

Interessenkonflikt

Die Autorinnen/Autoren geben an, dass kein Interessenkonflikt besteht.

\section{Autorinnen/Autoren}

\section{Tobias Bertsch, Gabriele Erbacher}

Europäisches Zentrum für Lymphologie, Földiklinik, Hinterzarten

\section{Korrespondenzadresse}

\section{Dr. Tobias Bertsch}

Europäisches Zentrum für Lymphologie, Földiklinik

Rösslehofweg 2-6

79856 Hinterzarten

Deutschland

tobias.bertsch@foeldiklinik.de

\section{Literatur}

[1] Abrufbar unter: https://www.thieme-con nect.com/products/ejournals/topten/ $10.1055 / \mathrm{s}-00034913$

[2] Bertsch T, Erbacher G, Corda D et al. Lipoedema - myths and facts, Part 5. European Best Practice of Lipoedema - Summary of the European Lipoedema Forum consensus. Phlebologie 2020; 49: 31-49

[3] Abrufbar unter: https://lipedema-simplified. org

[4] Abrufbar unter: https://www.fatdisorders. org

[5] Abrufbar unter: https://www.lipedema.org

[6] Abrufbar unter: https://www.fatdisorders. org/leadership-team

[7] Abrufbar unter: https://www.lipedema.org/ about/\#anchor-who

[8] Abrufbar unter: https://globalgenes.org/ event/fdrs-2020-focus-on-fat-disorders/

[9] Abrufbar unter: https://www.lipedema.org/ biobank-imaging-patient-treat-uofaarizona-herbst-research-grant

[10] Abrufbar unter: http://www.curelipedema. org/treat

[11] Abrufbar unter: https://www.lipedema.org/ patient-centered-research

[12] AL-Ghadban S, Cromer W, Allen M et al. Dilated Blood and Lymphatic Microvessels, Angiogenesis, Increased Macrophages, and Adipocyte Hypertrophy in Lipedema Thigh Skin and Fat Tissue. Journal of Obesity 2019; 2019: 4-10

[13] Felmerer G, Hägerling R, Gousopoulos E et al. Adipose Tissue Hypertrophy, An Aberrant Biochemical Profile and Distinct Gene Expression in Lipedema. J Surg Res 2020; 253: 294-303

[14] Gousopoulos E. Absence of lymphatic morphological changes but a distinct biochemical niche underlie lipedema development. Vortrag auf der Lymphologie 2019 in Bad Krozingen. Abstractband S. 21

[15] Abrufbar unter: https://www.lipedema.com/ lipedema-is-not-just-fat

[16] Abrufbar unter: https://www.lipedema.com/ us-lipedema-soc/\#join

[17] Abrufbar unter: https://www.lipedema.com/ about

[18] https://www.amazon.de/LymphedemaLipedema-Nutrition-Guide-supplements/dp/ 0976480689

[19] Abrufbar unter: https://www.pschyrembel. de/Ödem/KOFKR

[20] Bertsch T, Erbacher G. Lipödem - Mythen und Fakten Teil 2. Phlebologie 2018; 47: 120-126

[21] Hirsch T, Schleinitz J, Faerber $G$ et al. Is the differential diagnosis of lipoedema by means of high-resolution ultrasonography possible? Phlebologie 2018; 47 (4): 182-187

[22] Cellina M, Gibelli D, Soresina M et al. Noncontrast MR Lymphography of lipedema. Magent Resonance Imaging 2020; 71: 115124 
[23] Reich-Schupke S, Altmeyer P, Stücker M. Thick legs - not always lipedema. J Dtsch Dermatol Ges 2013; 11 (3): 225-233

[24] Lipedema - Guidelines in the Netherlands 2014. Abrufbar unter https://www.gdlymph. eu/assets/pdf/Dutch-lipoedema-guideline2014.pdf

[25] Bertsch T, Erbacher G, Corda D et al. Lipoedema - myths and facts, Part 5. European Best Practice of Lipoedema - Summary of the European Lipoedema Forum consensus. Phlebologie 2020; 49: 31-49

[26] Abrufbar unter: https://www.awmf.org/ leitlinien/detail/II/058-001.html

[27] Herpertz U. Das Lipödem. Lymphologie 1995; 19: 1-7

[28] Child AH, Gordon KD, Sharpe P et al. Lipedema: An inherited condition. Am J Med Genet Part A 2010; 152A: 970-976

[29] Erbacher G, Bertsch T. Lipoedema and pain: what is the role of the psyche? Results of a pilot study with 150 lipedema patients. Phlebologie 2020; 49: 305-316. doi:10.1055/a-1238-6657

[30] Dudek JE, Białaszek W, Ostaszewski P et al. Depression and appearance-related distress in functioning with lipedema. Psychology, health \& medicine 2018; 23 (7): 846-853

[31] Dudek JE, Bialaszek W, Ostaszewski P. Quality of life in women with lipoedema: a contextual behavioral approach. Quality of Life Research 2016; 25: 401-408

[32] Bosman J. Lipoedema: Poor knowledge, neglect or disinterest? Journal of Lymphoedema 2011; 6 (2): 109-111

[33] Bertsch T, Erbacher G. Lipödem - Mythen und Fakten Teil 3. Phlebologie 2018; 47: 188-197

[34] Bertsch T. Adipositas-assoziierte Lymphödeme - unterschätzt und unterbehandelt. Phlebologie 2018; 47: 75-83

[35] Mehrara B, Greene A. Lymphedema and Obesity: Is There a Link? Plast Reconstr Surg 2014; 134 (1): 154-160

[36] Ma W, Gil H], Escobedo N et al. Platelet factor 4 is a biomarker for lymphatic-promoted disorders. JCI Insight 2020; 5 (13): e135109. Published 2020 Jul 9. doi:10.1172/ jci.insight. 135109

[37] Lipedema simplified, webinar: Abrufbar unter. https://lipedema-simplified.org/ webinar2020-pf4/ Hier Minute 14:37 bis 14:47. Videosequenz kann aber auch beim Erstautor (Bertsch) angefragt werden

[38] Harwood CA, Bull RH, Evan J et al. Lymphatic and venous function in lipoedema. $\mathrm{Br}$ J Dermatol 1996; 134 (1): 1-6

[39] Bräutigam P, Földi E, Schaiper T et al. Analysis of lymphatic drainage in various forms of leg edema using two compartment lymphoscintigraphy. Lymphology 1998; 31 (2): 43-55

[40] Amann-Vesti BT. Druckmessung in den initialen Lymphgefäßen der Haut bei Patienten mit Lipödem. LymphForsch 2002; 6 (1): 7-9

[41] Buchwald H, Estok R, Fahrbach K et al. Weight and type 2 diabetes after bariatric surgery: systematic review and metaanalysis. Am J Med 2009; 122: 248-256

[42] Wittgrove AC, Clark GW. Laparoscopic Gastric Bypass, Roux en-Y - 500 Patients: Technique and Results, with 3-60 month follow-up. OBES SURG 2000; 10: 233

[43] Sugerman HJ et al. Diabetes and hypertension in severe obesity and effects of gastric bypass-induced weight loss. Ann Surg 2003; 237: 751-756

[44] Rasheid S, Banasiak M, Lipska A et al. Bypass is an Effective Treatment for Obstructive Sleep Apnea in Patients with Clinically Significant Obesity. OBES SURG 2003; 13: 5

[45] Courcoulas AP, Yanovski S, Arterburn DE et al. Long-term Outcomes of Bariatric Surgery: A National Institutes of Health Symposium. JAMA Surg 2014; 149 (12): 1323-1329

[46] Arterburn DE, Olsen M, Maciejewski M et al. Association Between Bariatric Surgery and Long-term Survival. JAMA 2015; 313 (1): 62-70

[47] Sjöström L, Nabro K, Sjöström D et al. Effects of bariatric surgery on mortality in Swedish obese subjects. N Engl J Med 2007; 357: 741-752

[48] Adams TD, Gress R, Hunt S et al. Long-term mortality after gastric bypass surgery. $\mathrm{N}$ Engl J Med 2007; 357: 753-761

[49] Shubeck S, Dimmick J, Telem D. Long-term Outcomes Following Bariatric Surgery. JAMA 2018; 319 (3): 302-303

[50] Fink JM, Schreiner L, Bertsch T et al. Leg Volume in Patients with Lipoedema following Bariatric Surgery. Visc Med 2020. doi:10.1159/000511044

[51] Linsmayer D, Neidlinger PK, Braus DF. Rheuma und Psyche - Eine Kurzübersicht. Orthopade 2019; 48 (11): 957-962. doi:10.1007/s00132-019-03812-8

[52] Baerwald C, Manger B, Hueber A. Depression als Komorbidität bei rheumatoider Arthritis. Z Rheumatol 2019; 78: 243-248 https://doi.org/10.1007/s00393-018-0568-5

[53] Hofmann P, Hemberger S, Lunzer R et al. Psychische Aspekte der rheumatoiden Arthritis Wie ansprechen? Wie behandeln? Zusammenfassung des Expertenmeetings „Rheuma trifft Psyche“ am 05.11.2015. Abrufbar unter: ttps://www.pfizermed.at/ sites/default/files/rheuma_trifft_psyche.pdf

[54] Bischoff N, Morina N, Egloff N. Chronischer Schmerz bei Traumatisierung. Komplexität und Herausforderung bei Diagnostik und Therapie. PiD - Psychotherapie im Dialog 2016; 17 (4): 69-72. doi:10.1055/s-0042116706

[55] Viana MC, Lim CCW, Pereira FG et al. Prior mental disorders and subsequent onset of chronic back or neck pain: findings from 19 countries. J Pain 2018; 19 (1): 99-110. doi:10.1016/j.jpain.2017.08.011

[56] Tegethoff M, Belardi A, Stalujanis E et al. Comorbidity of Mental Disorders and Chronic Pain: Chronology of Onset in Adolescents of a National Representative Cohort. The
Journal of Pain 2015; 16 (10): 1054-1064. doi:https://doi.org/10.1016/j.jpain. 2015.06.009

[57] Hooten WM. Chronic Pain and Mental Health Disorders. 2016 Mayo Foundation for Medical Education and Research, Mayo Clin Proc 2016; 91(7): 955-970. Abrufbar unter: https://www.mayoclinicproceedings.org/ article/S0025-6196(16)30182-3/pdf

[58] Jacobi F, Höfler M, Siegert J et al. Twelvemonth prevalence, comorbidity and correlates of mental disorders in Germany: The Mental Health Module of the German Health Interview and Examination Survey for Adults (DEGS1-MH). Int J Methods Psychiatr Res 2014; 23 (3): 304-319

[59] Deutsches Ärzteblatt Dienstag, 24. September 2019. Abrufbar unter: https://www. aerzteblatt.de/nachrichten/106211/ Betroffene-enttaeuscht-ueber-G-BAEntscheidung-zur-Liposuktion

[60] Gemeinsame Presseerklärung der organisierten Selbsthilfe von Frauen mit Lipödem vom 20.9.2019. Abrufbar unter: https:// webcache.googleusercontent.com/search? q=cache:gnx6Dnkwr1s]:https://www. lipoedem-fakten.de/app/download/ 6021579766/Gemeinsame\%2BPresseerkl\% 25C3\%25A4rung\%2BLip\%25C3\%25B6 dembetroffener\%2Bvom\%2B20.09.2019.pdf \%3Ft\%3D1579150244+\&cd=1\&hl= de\&ct=clnk\&gl=de\&client=safari

[61] Schmeller W. Letter to the Editor: Zu den drei Beiträgen: T. Bertsch und G. Erbacher: Lipödem - Mythen und Fakten Teil 1 bis 3. Phlebologie 2018; 47: 376-379

[62] Bertsch T, Erbacher G. Stellungnahme der Autoren auf Letter to the Editor von Schmeller W. Phlebologie 2018; 47: 379-384

[63] Abrufbar unter: https://www.lipedema.com/ us-lipedema-soc/\#supporters

[64] Abrufbar unter: https://www.lipedema.org/ namri-mri-crescenzi-donahue-researchgrant

[65] https://www.lipedema.org/hyaluronanextracellular-matrix-harten-research-grant/

\section{Publikationshinweis}

Leserbriefe stellen nicht unbedingt die Meinung von Herausgebern oder Verlag dar. Herausgeber und Verlag behalten sich vor, Leserbriefe nicht, gekürzt oder in Auszügen zu veröffentlichen.

Bibliografie

Phlebologie 2021; 50: 7-11

Online-Publikation: 1.10.2020

DOI $10.1055 / a-1250-3334$

ISSN 0939-978X

(C) 2020. Thieme. All rights reserved.

Georg Thieme Verlag KG, Rüdigerstraße 14,

70469 Stuttgart, Germany 